The Universe of Dwarf Galaxies

Editors : will be set by the publisher

EAS Publications Series, Vol. ?, 2021

\title{
THE MORPHOLOGICAL ORIGIN OF DWARF GALAXIES
}

\author{
Gerhard Hensler ${ }^{1}$
}

\begin{abstract}
Dwarf galaxies (DGs) serve as extremely challenging objects in extragalactic astrophysics. Their origin is expected to be set as the first units in CDM cosmology. Nevertheless they are the galaxy type most sensitive to environmental influences and their division into multiple types with various properties have invoked the picture of their variant morphological transformations. Detailed observations reveal characteristics which allow to deduce the evolutionary paths and to witness how the environment has affected the evolution. Here we refer to general morphological DG types and review some general processes, most of which deplete gas-rich irregular DGs. Moreover, the variety of pecularities is briefly refered, but cannot be comprehensively analyzed because of limited paper space.
\end{abstract}

\section{Introduction}

After a former conference talk on evolutionary models of dwarf galaxies (DGs), in particular, on dwarf irregular galaxies (dIrrs), the blue compact subclass (BCDs) and on starburst (SB)DGs, a senior colleague well-known as one of the pioneers of DG studies asked me with a serious surprise, why DG evolution is so complex and not a straight-forward scenario although their structural appearance looks pretty simple. Nowadays this first impression of DGs has changed totally with the advent of large telescopes, new techniques, and of the accessible panchromatic view. Within the last two decades detailed analyses of DGs has extended but also stirred our view of the classical morphological DG types, dIrrs, SBDGs, and dEs including satellite galaxies, so-called dwarf spheroidals (dSphs), as their extension to lower brightness of about $-5^{m}$.

In their studies of Virgo cluster DGs already Sandage \& Binggeli (1984) found that elliptical DGs (dEs) dominate the cluster galaxy population by far. This stands in contrast to their number fraction in the field where dIrrs are the most

\footnotetext{
1 University of Vienna, Institute of Astronomy, Tuerkenschanzst. 17, 1180 Vienna, Austria;
}

e-mail: gerhard.hensler@univie.ac.at 
The Universe of Dwarf Galaxies

frequent DGs but with a smaller fraction than cluster dEs (Binggeli et al. 1988). For the interpretation of this issue and the occurrence of enhanced SF in dIrrs Sandage \& Binggeli (1984) emphasized already the necessity of various links between the DG types by morphological transitions.

From the $\Lambda \mathrm{CDM}$ cosmology the baryonic matter should settle within Dark Matter (DM) halos which are originally prefered to form low-mass units, so-called subhalos, and hierarchically accumulating to massive galaxies. If the baryonic matter would follow this bottom-up structure formation, the subhalos should also assemble their gas at first and by this also evolve with star fomation (SF) to become the oldest galactic objects in the universe. That this picture seems to be too naive is simply understandable by three major physical principles: the first one is, that the gas assembly works on the free-fall timescale $\tau_{\mathrm{ff}}$, namely, dependent on the gas density as $\rho_{\mathrm{g}}^{-1 / 2}$, because gas is accreted through gravitation. At second, the SF timescale $\tau_{\mathrm{SF}}$ is per definition proportional to $\mathrm{M}_{g} / \Psi$ with $\Psi$ as the SF rate that, on the other hand, in the self-regulated SF mode depends on $\rho_{\mathrm{g}}^{2}$ (Koeppen, Theis, \& Hensler 1995). Since lower galaxy masses lead to less dense gas, SF is stretched over time for low-mass galaxies. And at least, as SF couples to stellar energy release and since the counteracting cooling process depends on

$\rho_{\mathrm{g}}^{2}$, the gas expands due to pressure support and reduces the SF rate so that the effect of SF self-regulation is non-linearly amplified.

Another important effect that seems to affect the whole network of galaxy formation and evolution is ionizing radiation from the first cosmic objects to the re-ionization of the gas in the universe. This changed not only its thermodynamical state so that its accretion onto low-mass objects was reduced (Dijkstra et al. 2004) but also evaporated gas that was already caught in minihalos (Barkana \& Loeb 1999). Since massive objects remained almost unaffected by the re-ionization phase, while DGs should have experienced delayed SF (Noeske et al. 2007), this evolutionary dichotomy is observed as downsizing (Cowie et al. 1996). Nevertheless, the assumption that all DGs were affected in the re-ionization era and in the same way would request overlapping Stroemgren bubbles in an almost uniformly ionized universe. This assumption, however, is questioned and contrasted by the existence and amplification of density structures (Partl et al. 2010).

\section{Dwarf irregular and dwarf starburst galaxies}

dIrrs are characterized by large gas fractions, ongoing SF, and low metallicities $Z$. That dIrrs consist of the same or a higher gas fraction than giant spiral galaxies and mostly suffer the same SF efficiency, but appear with a wide range, but lower $Z$ than spirals, cannot be explained by simple evolutionary models. When gas is consumed by astration but replenished partly by metal-enriched stellar mass loss, the general analytical derivation relates the element enhancement with the logarithm of the decreasing remaining gas content where the slope is determined by the stellar yields (see e.g. textbooks like by Pagel (2010) or reviews as e.g. by Hensler \& Recchi (2010)). The effective yields of gas-rich galaxies decrease, however, with smaller galaxy masses (Garnett 2002, van Zee 2001). This means 
that their element abundances, particularly $\mathrm{O}$ measured in HII regions, are much smaller than those released by a stellar population and confined to a "closed box".

Two processes can reduce the metal abundances in the presence of old stellar populations: loss of metal-enriched gas by galactic outflows or infall of metal-poor to even pristine intergalactic gas (IGM). It is widely believed, that a fundamental role in the chemical evolution of dIrrs is played by galactic winds, because freshly produced metals in energetic events are carried out from a shallow potential well of DGs through a wind (which will be therefore metal-enhanced). Some SBDGs are in fact characterized by galactic winds (Marlowe et al. 1995) or by large expanding supernova type II (SNeII)-driven X-ray plumes (e.g. Hensler et al. 1998, Martin et al. 2002). Studies have raised doubts to whether the expanding $\mathrm{H} \alpha$ loops, arcs, and shells mostly engulfing the X-ray plumes, lead really to gas expulsion from the galaxies because their velocities are mostly close to escape, but adiabatic expansion against external gas tends to hamper this.

As an extreme, Babul \& Rees (1992) speculated that galactic winds are able to empty DGs from its fuel for subsequent SF events and, by this, transform a gas-rich dIrr to a fading gas-poor system. In order to manifest this scenario and to study mass and element abundance losses through galactic winds numerous numerical models are performed under various, but mostly uncertain conditions and with several simplifications (e.g. MacLow \& Ferrara 1999, Strickland et al. 2004). The frequently cited set of models by MacLow \& Ferrara (self-gravitating, rotationally supported, isothermal HI disks of dIrrs with fixed structural relations for four different gas masses between $\mathrm{M}_{\mathrm{g}}=10^{6}-10^{9} M_{\odot}$ and three different SNII luminosities in the center corresponding to SN rates of one per $3 \times 10^{4}$ yrs to 3 Myrs) is mostly misinterpreted: The hot gas is extremely collimated from the center along the polar axis, but cannot sweep-up sufficient surrounding ISM to produce significant galactic mass loss. On the other hand, the loss of freshly released elements from massive stars is extremely high. Moreover, these models lack of realistic physical conditions, as e.g. the existence of an external pressure, self-consistent SF rates, a multi-phase ISM, etc.

Also more detailed numerical simulations (D'Ercole \& Brighenti 1999; Recchi \& Hensler 2006), show that galactic winds are not very effective in removing gas from a galaxy. Although galactic winds develop vertically, while the horizontal transport along the disk is very limited, their efficiency depends very sensibly on the galaxy structure and ISM properties, as e.g. on the HI disk shape (Recchi et al. 2009). Fig. 1 reveals clearly that the more eccentric the disk is, the more pronounced does the superbubble expand. On the one hand, the hot SN gas has to act against the galactic ISM, exciting turbulence and mixing between the metal-rich hot with the surrounding HI gas. Not taken into account in present-day models is the porosity of the ISM, consisting of clouds and diffuse less dense gas. In particular, the presence of clouds can hamper the development of galactic winds through their evaporation. This so-called mass loading reduces the wind momentum and internal energy. Since the metallicity in those clouds are presumably lower than the hot SNII gas, also the abundances in the outflow are diminished as e.g. observed in the galactic X-ray outflow of NGC 1569 (Martin et al. 2002) for which a mass-loading 

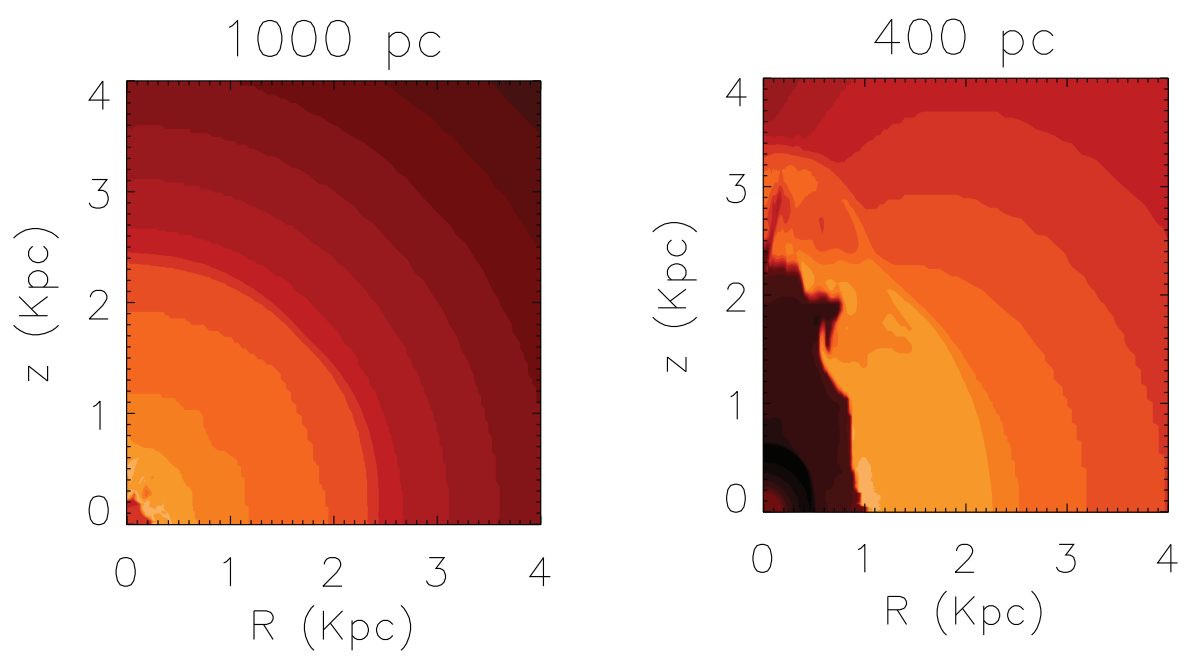

Fig. 1. Density contours after $200 \mathrm{Myr}$ of evolution for models differing on the semi-minor axis b of their initial configurations (semi-minor axis indicated on top of each panel), while the major axis is set to $a=1 \mathrm{kpc}$ : left: $\mathrm{b}=\mathrm{a}=1 \mathrm{kpc}$ and spherical symmetric; right: $\mathrm{b}=400 \mathrm{pc}$, i.e. a disk eccentricity amounts to $(\mathrm{a}-\mathrm{b}) / \mathrm{a}=0.6$. The density scale reaches from $10^{-27}$ (black) to $10^{-24} \mathrm{~g} / \mathrm{cm}^{3}$ (brightest). (for details see Recchi et al. 2009.)

factor of 10 is derived to reduce the metallicity to 1-2 times solar. In recent simulations Scannapieco \& Brueggen (2010) demonstrate that turbulent mixing can effectively drive a galactic wind. Although they stated that their models lead to a complex, chaotic distribution of bubbles, loops and filaments as observed in NGC 1569, other observational facts have not been compared.

Detailed numerical simulations of the chemical evolution of these SBDG by Recchi et al. (2006), e.g., could simultaneously reproduce both, the oxygen abundance in the warm gas as well as the metallicity in the hot outflow. Recchi \& Hensler (2007) show that the leakage of metals from a SBDG is surprisingly not prevented by the presence of clouds, because they pierce holes into the wind shells. This leads to a final metallicity of only a few tenths of dex lower than in models without clouds.

Consequently, the basic question must be answered which physical processes trigger such enormous SF rates as observed in SBDGs and would consume all the gas content within much less than the Hubble time. One possibility which has been favoured until almost two decades ago was that at least some of these objects are forming stars nowadays for the very first time. Today it is evident that even the most metal-poor ones (like I Zw 18) contain stars of at least 1 Gyr old (Momany et al. 2005), but most SBDGs have several Gyrs old stellar populations. This means that SF in the past should have proceeded in dIrrs, albeit at a low intensity what can at best explain their chemical characteristics, like for instance 
the low $[\alpha / \mathrm{Fe}]$ ratio.

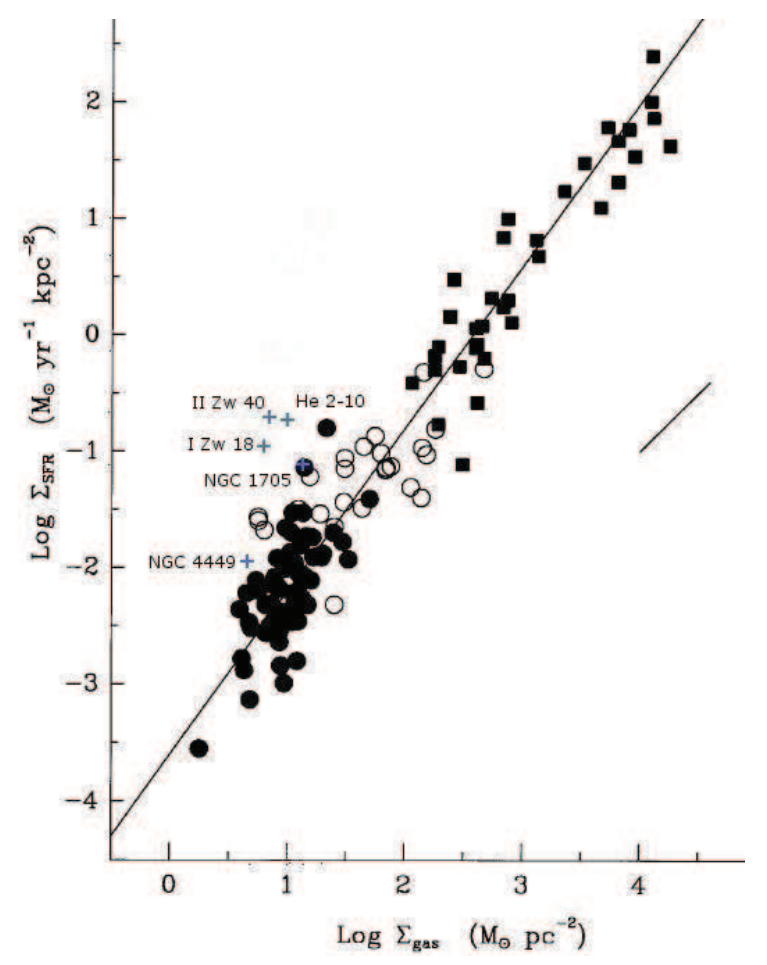

Fig. 2. Comparison of the star-formation rates vs. gas content, in the optical galactic body of a few prototypical starburst dwarf galaxies denoted by crosses and names with the well-known Kennicutt-Schmidt relation derived by Kennicutt (1998) with an exponent of 1.4 (long full-drawn line). (from Kuehtreiber 2011)

In most SBDGs large Hi reservoirs, however, enveloping the luminous galactic body have been detected (NGC 1705: Meurer et al. 1998, NGC 4449: Hunter et al. 1998, NGC 4569: Stil \& Isreal 2002, NGC 5253: Kobulnicky \& Skillman 2008, I Zw 18: van Zee et al. 1998c, II Zw 40: van Zee et al. 1998b) with clearly disturbed gas kinematics and disjunct from the luminous galactic body. Nevertheless, in not more than two objects, NGC 4569 (Mühle et al. 2005) and NGC 5253 (Kobulnicky \& Skillman 2008) gas infall is proven, while for the other cases the gas kinematics obtrudes that the gas reservoir feeds the engulfed DGs. In another object, He 2-10, the direct collision of an intergalactic gas cloud with a DG (Kobulnicky et al. 1995) is obviously triggering a huge SB.

Yet it is not clear, what happens to dIrrs if they experience on an increasing external pressure what would happen e.g. when they fall into galaxy clusters. In sect 3 we will discuss the effect of ram pressure on the structure of the ISM for which numerical models exist for spiral galaxies (e.g. Roediger \& Hensler 2005) as well as for dIrrs (e.g. Mori \& Burkert 2000), but only hints from observations. The effect on the SF rate due to compression of the ISM is observed, but not yet fully understood. Cortese et al. (2006) e.g. observed a coherent enhancement of SF in group galaxies falling into a cluster, therefore, denoted as blue infalling group. 
The $[\alpha / \mathrm{Fe}]$ vs. $[\mathrm{Fe} / \mathrm{H}]$ behaviour is representative of the different production phases, $\alpha$-elements from the short-living massive stars and iron to $2 / 3$ from type Ia SNe of longer-living binary systems. If the SF duration in a galaxy is very short, type Ia SNe do not have sufficient time to enhance the ISM with Fe and most of the stars will be overabundant in $[\alpha / \mathrm{Fe}]$. The low average $[\alpha / \mathrm{Fe}]$ ratios in dIrrs, however, compared to large galaxies serve as a hint of a long-lasting mild SF in these galaxies (Lanfranchi \& Matteucci 2004).

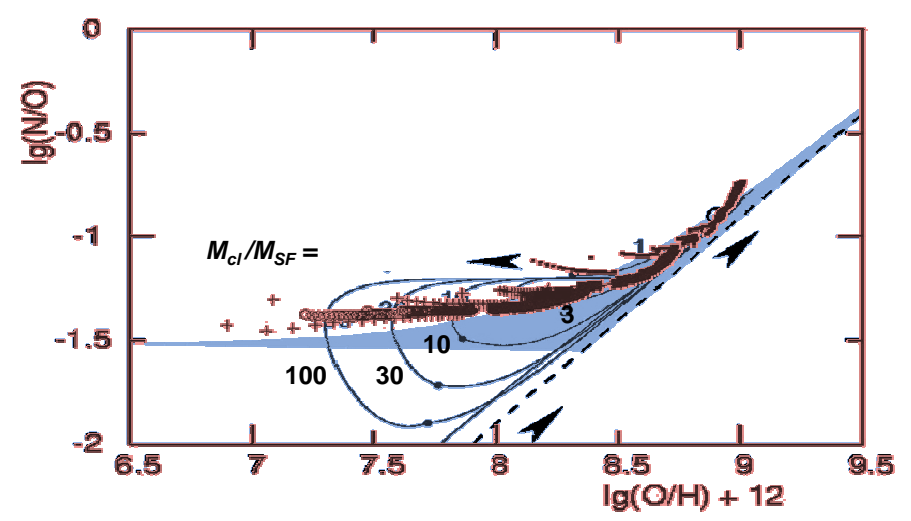

Fig. 3. The abundance ratio $\mathrm{N} / \mathrm{O}$ as a function of oxygen abundance observed in spiral and irregular galaxies (shaded area; after: van Zee et al. 1998a) overlayed with evolutionary loops due to infall of primordial intergalactic gas clouds. These have different mass fractions $M_{c l} / M_{S F}$ with respect to the mass involved in the SF region The crosses represent evolutionary timesteps of models, the arrows depict the direction of the evolutionary paths. The dashed straight line represents a simple model relation for purely secondary nitrogen production. For discussion see: Koeppen \& Hensler 2005).

Although the mass-metallicity relation also holds for dIrrs and even steepens its slope (Trimonti et al. 2004) what can be interpreted by galactic mass loss and the corresponding lower effective yield (Garnett 2002), the abundance ratios are unusual. As mentioned above $\mathrm{O} / \mathrm{Fe}$ reaches already solar values for subsolar oxygen abundances. While this can be explained by means of a long SF timescale, another characteristic and unusual signature is that with $\mathrm{O}$ abundances below 1/10 solar the $\mathrm{N} / \mathrm{O}$ ratio $\log (\mathrm{N} / \mathrm{O})$ remains at about -1.6 , i.e. clearly smaller than in $\mathrm{gSs}$ and with a large scatter but no significant correlation with oxygen (see Fig 3). Their regime of $\mathrm{N} / \mathrm{O}-\mathrm{O} / \mathrm{H}$ values overlaps with those of $\mathrm{HII}$ regions in the outermost disk parts of gSs at around $12+\log (\mathrm{O} / \mathrm{H})=8.0 \ldots 8.5$ (van Zee et al. 1998a).

In the 90 th several authors have tried to model these observations by SF variations with gas loss through galactic winds under the assumptions that these dIrrs and BCDGs are young and experience their first epochs of SF (for a detailed review see Hensler et al. 1999). Since stellar population studies contradict to the youth hypothesis, another process must be invoked. Since these objects 
are embedded into $\mathrm{Hi}$ envelops and are suggested to suffer gas infall as manifested e.g. for NGC 1569 (see above, Stil \& Isreal 2002, Mühle et al. 2005), the influence of metal-poor gas infall into an old galaxy with continuous SF on particular abundance patterns should be exploited. With the reasonable approach that the fraction of infalling gas increases with decreasing galaxy mass, their results could match not only the observational regime of BCDs in the $[12+\log (\mathrm{O} / \mathrm{H})$ ]- $\log (\mathrm{N} / \mathrm{O})$ space but also explain the shark-fin shape of observational data distribution (Koeppen \& Hensler 2005).

\section{Dwarf elliptical galaxies}

dEs are the most numerous type in clusters and are frequently denoted as examples of "stellar fossil" systems in which the bulk of their SF occurred in the past. They are preferentially located in morphologically evolved environments (Trentham \& Tully 2002), i.e. in regions with high galaxy densities and dominate the morphological types of galaxy in galaxy clusters, as e.g. Virgo, Coma, Fornax, and Perseus. Furthermore, Tully \& Trentham (2008) observed that dEs strongly cluster around luminous elliptical/S0 galaxies. The evolution of this galaxy type should be mainly caused by gas and tidal effects on SF and structure and indicates that it is strongly affected by environment.

Already Bothun et al. (1985) found that cluster dEs are usually almost free of interstellar gas and contain few young stars. In trying to understand the dE population, structural regularities and correlations must be studied, as it is known since the 80th between optical surface brightness and luminosity (Binggeli et al. 1984, Kormendy 1985) and between luminosity and stellar velocity dispersion which also correlate with metallicity (e.g. Petersen \& Caldwell 1993). Furthermore, dEs often have flattened profiles but are mostly kinematically supported by their stellar velocity dispersions rather than by rotation (Bender et al. 1991).

The combination of low gas-mass fractions and moderate-to-low stellar metallicities in $\mathrm{dE}$ (about 0.1 of solar or less) is a key feature of this class. The lower abundances of stars in dEs (Han et al. 1997) suggest that extensive gas loss occurred during their evolution and SF ceased due to a lack of raw material rather than exhaustion of the gas supply through SF. Galactic winds are therefore a hallmark of modern models for $\mathrm{dE}$ galaxies, starting from the basic consideration by Larson (1974) and continued with the study by Dekel \& Silk (1986). They are commonly assumed to have cleaned out $\mathrm{dE}$ galaxies soon after their formation. As mentioned in sect. 2 gas expulsion by means of galactic winds even in low-mass systems requires a dark-to-baryonic matter ratio (MacLow \& Ferrara 1999) much smaller than assigned to DGs in the classical formation picture (e.g. Mateo 1998). There are two competing scenarios for the formation of dEs. On the one hand, those low-mass galaxies are believed to constitute the building blocks in $\Lambda \mathrm{CDM}$ cosmology and should therefore have evolved congruently with the mass accumulation to the much more massive entities, galaxies and galaxy clusters, by this leading to SF with the downsizing effect through the delay by the re-ionization epoch. Their stellar component is expected to be heated continuously by harras- 
ment of more massive cluster galaxies and thus to be pressure supported.

A variety of observations are available which also support diverse scenarios of dEs evolution (see e.g. review by Lisker, conference). Recent HI studies of Virgo cluster dEs (Conselice et al. 2003) and also those of the Fornax cluster (e.g. Michielsen et al. 2004) have unveiled that a small but significant fraction of them contains gas, has experienced recent SF, and can be argued from internal kinematics and cluster distribution data to represent an infalling class of different types of gas-rich galaxies in the state of morphological transformation. Recent findings of a significant fraction of rotationally supported dEs in the Virgo cluster (van Zee et al. 2004) also supports the possibility of morphological transformation from dIrrs to dEs thru gas exhaustion (Boselli et al. 2008). This separation should therefore also be visible in an intermediate age stellar population, flatter figure shape, and rotation. Indeed, Lisker et al. (2007) found that dEs in the Virgo cluster can be divided in different subclasses which differ significantly in their morphology and clustering properties, however do not show any central clustering, but are distributed more like the late-type galaxies. These subclasses show different disk signatures, such as bars and spiral structures. Also these types of dEs are not spheroidal, but rather thick disk-like galaxies.

Similar shapes were also found for the brighter, non-nucleated dEs. There is only a small fraction of nucleated dEs whithout any disk features or cores, which keep the image of spheroidal objects consisting of old stars.

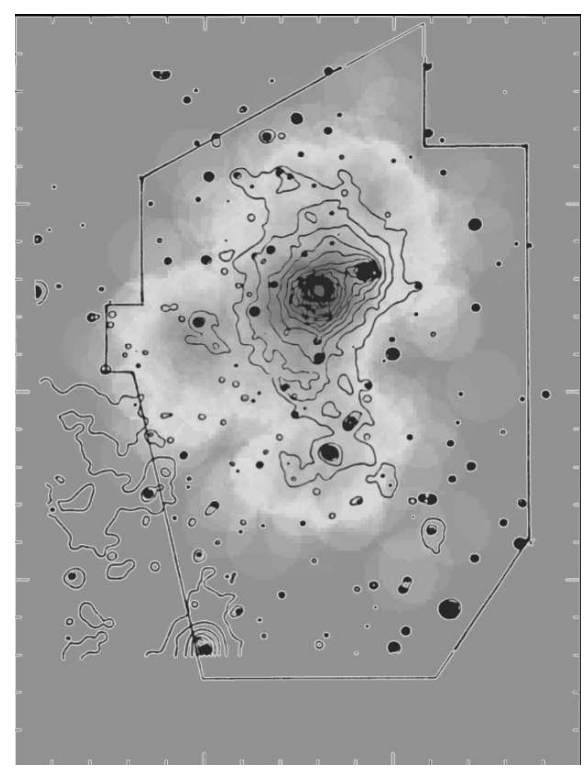

Fig. 4. Distribution of dEs in the Virgo Cluster divided into rapid (white) and slow objects (darker central part) overlaid with X-Ray brightness contours (courtesy by Thorsten Lisker).

A figure analysis of Virgo dEs correlates with the averaged orbit velocity in the sense that flatter (infalling and transformed) dEs show on average a larger or- 
bital velocity $(700 \mathrm{~km} / \mathrm{s})$ than those originating within the cluster $(300 \mathrm{~km} / \mathrm{s})$ (Lisker et al. 2009). This kinematical dichotomy is expected because galaxies formed in virial equilibrium with the cluster retain their initial kinetic energy while the cluster mass grows. Galaxies falling into the present cluster potential must therefore obtain larger velocities.

To obtain information about their both evolutionary stages, the young infalling vs. the late cluster members, Gotthart et al. (2009) studied SDSS data. The basic model is that dIrrs which are formed outside the Virgo Cluster and becoming stripped on their infall, by this being transformed into dEs, should reveal properties recognizably different from dEs which have already aged in the cluster, as e.g. colors, effective radius, radial stellar distribution, and abundances. One result by Gotthart et al. (2009) is that for the two dE populations, with and without cores, distinguished by their Sersic parameter, there is only a slight indication that nonnucleated dEs are more concentrated towards the inner cluster regions, whereas the fraction of nuclated dE is randomly distributed, while Lisker et al. (2007) found it to increase with distance. An analysis of the relation between the central surface brightness and the Sersic parameter shows the expected tendency to higher values for brighter galaxies. Furthermore, there were no relations found for the Sersic parameter, the effective radius, or the distance from M87.

For deeper insights spectra are urgent. And really, Toloba et al. (2009) derived for Coma cluster dEs to be weaker in carbon than dEs in low-density environments, while they are similar in nitrogen. Most recently, Toloba et al. (2010) found that pressure supported Virgo dEs show higher dynamical mass-to-light ratios than rotationally supported dEs of similar luminosity and further that dEs in the outer parts of the cluster are mostly rotationally supported with disky shapes.

Rotationally supported dEs even follow the Tully-Fisher relation. One of Toloba et al. (2010) fundamental and most spectacular results is, however, that dEs are not DM-dominated galaxies, at least out to the half-light radius. Concerning any metallicity gradient, the picture is not yet clear, but also not what one should expect. While Spolaor et al. (2009) show a tight positive correlation between the total metallicity $Z$ and the mass, Koleva et al. (2009) do not find any trend involving $[\mathrm{Fe} / \mathrm{H}]$ for Fornax-cluster and nearby-group dEs. However, they found metallicity gradients to exist and argue that this is a lack of sufficient mixing of old stellar populations but compare it also with simulations which found this as a result of galactic winds. Moreover, from the deconvolution of the SF history of their sample dEs with respect to the central 1 arcmin and within the effective radius Toloba et al. (2009) allow the conclusion on the existence of SF episodes in the very center even within the last 1 Gyr for a few objects. Correlations of both signatures, SF history and metallicity gradients, for cluster-member dEs vs. infall dEs should be derived for more clusters, but observations are unfortunately very time-expensive if possible at all. 


\section{Dwarf spheroidal galaxies}

At the faint end of dEs another type of almost gas-free spheroidal DGs exists which is located around massive galaxies like our MWG and M31 and thus orbiting them as satellites. These objects have attracted increasing attention over the last years because at their low-mass end they overlap with Globular Clusters, so that the understanding of their formation and evolution is of substantial relevance for our astrophysical picture of cosmology and galaxy evolution. Four main questions are addressed:

1. How and when did they form? They all harbour a very old stellar population (Tolstoy et al. 2009) and seem to have evolved unaffected of the re-ionization era (Grebel \& Gallagher 2004).

2. Is their existence as satellite system typical for all massive galaxies? Their origin and DM content is still questioned by some authors (Kroupa et al. 2010). The large discrepancy of the number of objects really observed and the one expected from $\Lambda \mathrm{CDM}$ cosmology and because of their orbit concentration to the so-called disk of satellites, also observed around M31, invoked the preferrence of their tidal-tail origin (Metz et al. 2009). The large velocity disperion is caused by the tidal effects.

3. How is their evolution determined by the vicinity of the massive mature galaxy? Not only the tidal field must have a disruptive effect, but also a gaseous halo of the central galaxy will interact with the ISM of the dSphs (Mayer et al. 2007). The tendency of an increasing gas fraction bound to the dSphs with distance from the MWG (Harbeck et al. 2001), points into that direction.

4. Vice versa the question arises, how the satellites influence the structure and evolution of the mature galaxy, here the MWG.

The first three questions also concern the morphological origin of dSphs e.g. as tidal-tail DGs formed in the same era as the MWG but in a gas-rich galaxy collision or their transition from gas-rich $\Lambda$ CDM satellites to dSphs.

The fascinating wealth of data and their precision on stellar ages and kinematics, on their chemical abundances, abundance gradients, and tidal tails of dSphs (for most recent reviews see e.g. Koch (2009) and Tolstoy et al. 2009) have triggered numerous numerical models which, however, still lack of the inclusion of the above-mentioned environmental effects. Not before recently Petrov \& Hensler (2011, first insights also in this conference proceedings and in Hensler (2011)) have started to simulate the full sample of a large number of DM subhalos containing baryonic matter by chemo-dynamical models. Since these models cannot be presented here because of paper space, we wish to highlight only the main issues as follows and refer the interested reader to a forth-coming paper by Petrov \& Hensler (2011). In addition to the tidal and gaseous effects by means of the MWG, due to the mutual interactions also between the dSphs disruption, gas and star exhaustion, and merging occur. The metallicity and abundance ratios are traced and comparable with observation. The SF histoies vary dependent on mass and gas re-accretion. 


\section{Acknowledgement}

The author is grateful to Joachim Koeppen, Thorsten Lisker, Mykola Petrov, Simone Recchi, Werner Zeilinger, and the SMAKCED collaborations for enlightening and continuous discussions on DGs.

\section{References}

Babul, A. \& Rees, M.J. 1992, MNRAS, 255, 346

Barkana, R. \& Loeb, A. 1999, ApJ, 523, 54

Bender, R., Paquet, A., \& Nieto, J.-L. 1991, A\&A, 246, 349

Binggeli, B., Sandage, A., \& Tarenghi, M. 1984, AJ, 89, 64

Binggeli, B., Sandage, A., \& Tammann, G.A. 1988, ARA\&A, 26, 509

Boselli, A., et al. 2008, ApJ, 674742

Bothun, G.D., et al. 1985, AJ, 90, 697

Conselice, C.J., et al. 2003 ApJ, 591, 167

Cortese, L., et al. 2006, A\&A, 453, 853

Cowie, L.L., et al. 1996, AJ, 112, 839

Dekel, A., \& Silk, J. 1986, ApJ, 303, 39

D'Ercole, A., \& Brighenti, F. 1999, MNRAS, 309, 941

Dijkstra, M., et al. 2004, ApJ, 601, 666

Garnett, D.R. 2002, ApJ, 581, 1019

Gotthart,T., et al. AN, 330, 1037

Grebel, E.K., \& Gallagher, J.S. 2004, ApJ, 610, L89

Grebel, E.K., Gallagher, J.S., \& Harbeck, D. 2003, AJ, 125, 1966

Han, M., et al. 1997, AJ, 113, 1001

Harbeck, D., et al. 2001, AJ, 123, 3092

Hensler, G. 2011, in: P. Papaderos, S. Recchi, \& G. Hensler (eds.), Dwarf Galaxies: Keys to Galaxy Formation and Evolution, Proc. JENAM 2010 Symp. No. 2, Springer Publisher, in press

Hensler, G. \& Recchi, S. 2010, in: K. Cunha, M. Spite \& B. Barbuy (eds.), Chemical Abundances in the Universe: Connecting First Stars to Planets, Proc. IAU Symp. No. 265, p. 325

Hensler, G., et al. 1998, ApJ, 502, L17

Hensler, G., Rieschick, A., \& Koeppen, J. 1999, in: J. Beckman \& T.J. Mahoney (eds.), The Evolution of galaxies on Cosmological Timescales, ASP Conf. Ser., 187, 214

Hunter, D.A., et al. 1998, ApJ, 495, L47

Johnston, K., et al. 2008, ApJ, 689, 936

Kennicutt, R.J. 1998, ApJ, 498, 541

Kobulnicky, H.A., \& Skillman, E.D. 2008, AJ, 135, 527

Kobulnicky, H.A., et al. 1995, AJ, 110, 1116

Koch, A. 2009, AN, 330, 675

Koeppen, J. \& Hensler, G. 2005, A\&A, 434, 531

Koeppen, J., Theis, C., \& Hensler, G. 1995, A\&A, 296, 99

Koleva, M., et al. 2009, MNRAS, 396, 2133 
Kormendy, J. 1985, ApJ, 295, 73

Kroupa, P., et al. 2010, A\&A, 523, A32

Kuehtreiber, M. 2011, Bachelor thesis, Univ. of Vienna

Lanfranchi, G.A., \& Matteucci, F. 2004, MNRAS, 351, 1338

Larson, R.B. 1974, MNRAS, 169, 229

Lisker, T., et al. 2007, ApJ, 660, 1186

Lisker, T., Janz, J., Hensler, G., et al. 2009, ApJ, 706, 124

MacLow, M.-M. \& Ferrara, A. 1999, ApJ, 513, 142

Marlowe, A.T., et al. 1995, ApJ, 438, 563

Martin, C.L., Kobulnicky, H.A., \& Heckman, T.M. 2002, ApJ, 574, 663

Mateo, M. 1998, ARA\&A, 36, 435

Mayer, L. et al. 2007, Nature, 445, 738

Metz, M., et al. 2009, ApJ, 697, 269

Meurer, G.R., Staveley-Smith,L., \& Killeen, N.E.B. 1998, MNRAS, 300, 705

Michielsen, D., et al. 2004, MNRAS, 353, 1293

Momany, Y., et al. 2005, A\&A, 439, 111

Mori, M. \& Burkert, A. 2000, ApJ, 538, 559

Mühle, S., et al. 2005, AJ, 130, 524

Noeske, K.G., et al. 2000, A\&A, 361, 33

Noeske, K.G., et al. 2007, ApJ, 660, L47

Pagel, B.E.J. 2010, Nucleosynthesis and galactic chemical evolution, (Cambridge Univ. Press)

Partl, A.M., et al. 2010, A\&A, 524, A85

Petersen, R.C., \& Caldwell, N. 1993, AJ, 105, 1411

Petrov, M., \& Hensler, G. 2011, ApJ, submitted

Read, J.I., et al. 2006, MNRAS, 366, 429

Recchi, S., \& Hensler, G. 2006, A\&A, 445, L39

Recchi, S., \& Hensler, G. 2007, A\&A, 476, 841

Recchi, S., Hensler, G., et al. 2006, A\&A, 445, 875

Recchi, S., Hensler, G., \& Anelli, D. 2009, arXiv:0901.1976

Roediger, E., \& Hensler, G. 2005, A\&A, 431, 85

Sandage, A. \& Binggeli, B. 1984, AJ, 89, 919

Scannapieco, E., \& Brueggen, M. 2010, MNRAS, 405, 1635

Spolaor, M., et al. 2009, ApJ, 691, L138

Stil, J. M., \& Israel, F. P. 2002, A\&A, 392, 473

Strickland, D.K., et al.. 2004, ApJ, 606, 829

Toloba, E., et al. 2009, ApJ, 707, L17

Toloba, E., et al. 2010, arXiv:1011.2198

Tolstoy, E., et al. 2009, ARA\&A, 47, 371

Trentham, N., \& Tully, R.B. 2002, MNRAS, 335, 712

Trimonti, C.A., et al. 2004, ApJ, 613,898

Tully, R.B., \& Trentham, N. 2008, AJ, 135, 1488 
van Zee, L. 2001, AJ, 121, 2003

van Zee, L., Salzer, J.J., \& Haynes, M.P. 1998a, ApJ, 497, L1

van Zee, L., Skillman, E.D., \& Salzer, J.J. 1998b, AJ, 116, 1186

van Zee, L., et al. 1998c, ApJ, 497, L1

van Zee, L., Skillman, E.D., \& Haynes, M.P. 2004, ApJ, 218, 211 